\title{
Profilaufbau, Molluskenführung und Parallelisierungsmöglichkeiten des Altwürmabschnitts im Löss- profil Mainz-Weisenau
}

\author{
Erhard Bibus, Wolfgang Rähle \& Joachim Wedel**)
}

Bibus, E., RäHLE, W. \& Wedel, J. (2002): Profilaufbau, Molluskenführung und Parallelisierungsmöglichkeiten des Altwürmabschnitts im Lössprofil Mainz-Weisenau. - Eiszeitalter und Gegenwart, 51: 1-14; Hannover 2002.

Keywords: Jungpleistozän, Löss, Paläoböden, Humuszonen, Mollusken, Paläoklima, Paläoökologie, Mainzer Becken.

Kurzfassung: In den Lössdeckschichten des Steinbruchs Mainz-Weisenau (Heidelberger Zement AG) sind im Hangenden des letztinterglazialen Bodens drei Altwürmhumuszonen aufgeschlossen (Mosbacher Humuszonen). Auf der Grundlage einer detaillierten Profilbeschreibung erfolgte eine lückenlose Entnahme von Sedimentproben zur Untersuchung der Molluskenfaunen. Die Aussage des Fossilberichts wird durch die Anwesenheit von verlagerten Sedimenten unter und zwischen den Humuszonen und die Möglichkeit einer sekundären Einmischung von Schalenmaterial beeinträchtigt. Dennoch lässt der Fossilbericht nach dem letzten Interglazial drei Wärmeschwankungen erkennen, die mit den Humuszonen korrelierbar sind. In den Sedimenten zwischen Eemboden und unterer Humuszone sowie zwischen mittlerer und oberer Humuszone wurden Reste kaltzeitlicher Faunen festgestellt, die auf Kälteperioden hinweisen. Der Bereich der unteren und, in geringerem Maße, der Bereich der mittleren Humuszone zeichnen sich durch die Anwesenheit von warmzeitlichen Faunenelementen aus (darunter ausgesprochene Waldarten), was auf einen mehr oder weniger hohen Grad an Wiederbewaldung schließen lässt. Es handelt sich dabei ausnahmslos um Waldarten, die sowohl in Laub- als auch in Nadelwaldbeständen auftreten können. Der Abschnitt zwischen unterer und mittlerer Humuszone und die obere Humuszone führen lediglich Steppen- und Waldsteppengesellschaften, die eng miteinander verzahnt sind. Der vorliegende Fossilbericht lässt sich gut mit den allgemein akzeptierten Vorstellungen über die Klimaentwicklung im frühen Würm vereinbaren, die sich aus Tiefsee- und Eisisotopenkurven ergeben haben. Zum gegenwärtigen Zeitpunkt wird eine Parallelisierung der unteren und mitt-

\footnotetext{
*)Anschriften der Verfasser: Prof. Dr. E. Bibus, Geographisches Institut der Universität Tübingen, Hölderlinstraße 12, D-72074 Tübingen, Dr. W. RÄHLE, Zoologisches Institut der Universität Tübingen, Auf der Morgenstelle 28, D-72076 Tübingen, J. WEDEL, Hessisches Landesamt für Umwelt und Geologie, Rheingaustraße 186, D-65203 Wiesbaden.
}

leren Humuszone von Mainz-Weisenau mit den beiden bewaldeten norddeutschen Interstadialen Amersfoort/ Brörup und Odderade sowie den beiden St-GermainInterstadialen in Ostfrankreich für wahrscheinlich gehalten. Für die Korrelation der oberen Humuszone werden verschiedene Möglichkeiten diskutiert.

\section{[Structural and malacozoological studies in the Early-Wuermian part of the loess section at Mainz-Weisenau (Western Germany), and possibilities for correlation]}

Abstract: In the covering loess layers of the quarry of Mainz-Weisenau (Heidelberger Zement AG), three Early Wuermian humic zones (Mosbacher Humuszonen) are exposed above the last interglacial soil. Based on a detailed description of the section, a complete series of samples was collected for malacozoological studies. The evidence of the fossil record is somewhat compromised by displaced sediments under and between the humic zones, and by the possibility of secondary introduction of shells. Nonetheless, the fossil record shows evidence for three warmer phases after the last interglacial, which are correlated with the humic zones. Remains of glacial index species were found in the deposits between the last interglacial soil and the lower humic zone, as well as in the deposits between the middle and the upper humic zone, which indicate that cold periods occurred. The lower and, on a minor scale, the middle humic zone display characteristic elements of warm phases (among them several dedicated woodland species), which indicate a certain extent of reforestation. Only woodland species could be detected which can live in both deciduous and coniferous forests. Between the lower and middle humic zone, as well as in the upper humic zone, only steppe and open woodland associations were found, which were closely linked together. The results of the present study are very consistent with the generally accepted ideas on the succession of climates during the Early Wuermian period, based on marine and ice core records. Currently, we believe that the lower and middle humic zones at MainzWeisenau correlate with the two wooded interstadials Amersfoort/Brörup and Odderade in Northern Germany, as well as the St. Germain interstadials in Eastern France. Differences of opinion remain regarding the correlation of the upper humic zone. 


\section{Einleitung}

In den verschiedenen Lössprovinzen von Deutschland ist im Altwürmabschnitt neben Solifluktionsdecken häufig eine der Mosbacher Humuszonen aufgeschlossen. Nur sehr selten sind bis zu drei als interstadial angesehene Humuszonen nachzuweisen (z.B. SchÖNHALs et al. 1964, SEMMEL 1968, Rohdenburg \& Meyer 1966). Solche Profile, insbesondere mit zwischengelagertem Löss, sind in Deutschland gegenwärtig nur im Rhein-Maingebiet zugänglich, wobei sich das Lössdeckschichtenprofil im Kalksteinbruch der Heidelberger Zement Aktiengesellschaft in Mainz-Weisenau als besonders aussagekräftig erwiesen hat. In dem Aufschluss ist von SEMMEL der voranschreitende Abbau seit zwei Jahrzehnten verfolgt und die Bedeutung des Profils für die paläopedologische Untergliederung des älteren Würmlösses mehrfach diskutiert worden (z.B. Semmel 1995, 1996a, 1999a). Zudem erfolgten in dem Aufschluss zahlreiche Spezialuntersuchungen, wie z.B. Dünnschliffanalysen, TL- und IRSL-Datierungen, gesteinsmagnetische Untersuchungen, Pollenanalysen und Molluskenbestimmungen (vgl. Einzelbeiträge in SEMMEL 1996a). Ziele dieser Untersuchungen waren stratigraphische, paläoklimatische, paläopedologische und paläoökologische Fragestellungen, wobei auch immer wieder das Problem angeschnitten wurde, in welcher Weise der Altwürm-Humuszonenkomplex mit Sauerstoffisotopenkurven oder mit der Gliederung in Norddeutschland und im Alpenvorland zu parallelisieren ist (vgl. BiBus et al. 1996, SEMMEL 1999a). Was die Molluskenführung des älteren Würmlösses anbetrifft, so sind bislang in Mainz-Weisenau nur Funde aus der obersten Humuszone bekannt geworden (vgl. RÄHLE in BiBUs et al. 1996: 22 ff.). In den letzten Jahren konnten nun erstmals auch im gesamten übrigen Altwürmbereich Schnecken nachgewiesen werden. Es sind dabei von SEMMEL und BiBus in den Jahren 1998 und 1999 mehrfach z.T. nahezu lückenlose Probeserien mit einer Menge bis zu 7 Liter pro Einzelprobe entnommen worden. Ihr Fauneninhalt wurde von RÄHLE und WEDEL bestimmt. Es bestand die Hoffnung, dass sich durch die Auswertung des gesamten Materials, über das von BludAU (in BiBus et al. 1996; vgl. Abb. 3) erarbeitete Pollendiagramm hinaus, zusätzliche paläoklimaökologische Informationen ergeben würden.

\section{Profilbeschreibung}

Im Steinbruch Mainz-Weisenau liegt der ältere Würmlöss in Dellenfüllungen, die sich in die älteren Lösse eingeschnitten haben. Im Zuge des Abbaus waren mehrfach entsprechende Dellensysteme mit ähnlichen Abfolgen aufgeschlossen. Die Ergebnisse von sedimentologischen und pollenanalytischen Untersuchungen aus einer solchen Hohlform (Delle 1) sind in Abbildung 2 und 3 wiedergegeben (nach BiBus et al. 1996). Die jetzt untersuchten, schneckenführenden Schichten liegen in einer angrenzenden Delle (Delle 2, Abbaustand 1998/99) im südlichsten Bereich des Abraumaufschlusses, der an dieser Stelle besonders tief in den Hangbereich eingreift. Die vollständigsten Profile treten im Dellentiefsten auf, während an den Dellenflanken die Abfolgen infolge starker Erosion erheblich reduziert sind oder vollkommen fehlen. Es ist deshalb damit zu rechnen, dass Material von der Seite in das Dellentiefste verlagert wurde, was sich auch in Bodendünnschliffen zeigt, die abgerollte Fragmente aus $\mathrm{Ah}$ und $C_{C}-$ Material enthalten (SEMmel 1999b: 60 und Foto 3).

An der Basis der beprobten Abfolge ist der bis 80 $\mathrm{cm}$ mächtige, rötlichbraune Rest eines $\mathrm{B}_{\mathrm{t}}$-Horizontes erhalten, der sich auf einem gelbbraunen Löss entwickelt hat. In diesem Löss ist der kräftige $C_{C^{-}}$ Horizont des Bodens mit Lösskindeln bis zu 10 cm Durchmesser ausgebildet (vgl. Abb. 1). Die Intensität des $\mathrm{B}_{\mathrm{t}}$-Horizontes spricht für eine interglaziale Bildung. Aufgrund der stratigraphischen Position und spärlich auftretenden, warmzeitlichen Mollusken handelt es sich mit großer Wahrscheinlichkeit um den Rest der letztinterglazialen $\mathrm{Pa}$ rabraunerde. Der $\mathrm{B}_{\mathrm{t}}$-Horizont ist im obersten Bereich durch Solifluktionsvorgänge verlagert. In einer kleinen Hohlform hat die Solifluktion auch tiefere Bereiche des Bodens erfasst. An anderer Stelle des Aufschlusses ist der Eem-Boden vollkommen erodiert, so dass dort direkt unter den altwürmzeitlichen Mosbacher Humuszonen die vorletztkaltzeitlichen Weilbacher Humuszonen liegen. Solche Diskordanzen müssen erkannt werden, um Fehlinterpretationen zu vermeiden (vgl. BiBus et al. 1996: 34). Im aufgenommenen Standardprofil umfasst die Diskordanz auf jeden Fall den gesamten $\mathrm{A}_{1}$-Horizont der letztinterglazialen Parabraunerde.

Über der Solifluktionsdecke liegt ein gelber, feinsandiger Schluff mit vereinzelt eingelagerten Tonschmitzen aus rötlichbraunem $\mathrm{B}_{\mathrm{t}}$-Substrat. Offen- 
in $\mathrm{m}$ : Probennr.

0

$1-$

$\prod_{14}^{14} 18$

$2-$

13

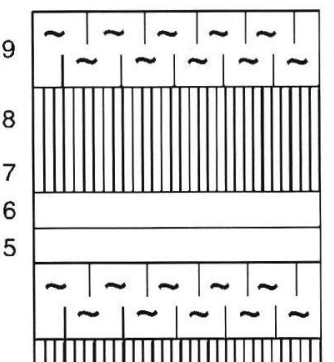

umgel. Humuszone

Humuszone, $\mathrm{OMHZ}$

Löß

Löß, hellbraun

umgel. Humuszone

Humuszone, $\mathrm{MMHZb}$

$\mathrm{B}_{\mathrm{V}}$-Horizont

12

$3-$

10

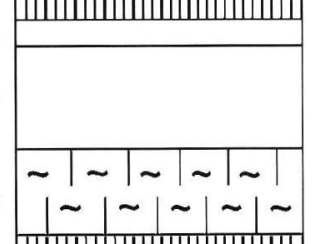

Humuszone, MMHZa

LÖß

LÖß, schwach humos

umgel. Humuszone

4 -

$5-$

$6-$

$7-$

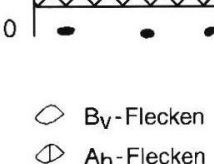

Abb. 1: Profilaufbau des molluskenführenden Altwürmabschnitts in Delle 2 von Mainz-Weisenau (Abbaustand 1998/99). Die Entnahmestellen der Molluskenproben sind von 0 bis 19 durchnummeriert. Probe $\mathrm{X}$ aus Tabelle 2 stammt aus einem anderen Profil (Delle 1) und entspricht etwa den Proben 17 und 18. OMHZ = obere Humuszone, $\mathrm{MMHZb}=$ mittlere Humuszone oben, $\mathrm{MMHZa}=$ mittlere Humuszone unten, $\mathrm{UMHZb}=$ untere Humuszone oben, $\mathrm{UMHZa}=$ untere Humuszone unten.

Fig. 1: Profile containing mollusca of the Lower Wuermian in the depression number 2 in Mainz-Weisenau (stage of exhaustion 1998/99). The sampling points of mollusca are numbered consecutively from 0 to 19 . Sample $\mathrm{X}$ in table 1 comes from another section (depression number 1), and corresponds approximetely to samples 17 and 18 . sichtlich handelt es sich um eine durch Verschwemmung entstandene Schicht aus hangaufwärts anstehenden Weisenauer Sanden und verlagertem $\mathrm{B}_{\mathrm{t}}$-Material. Über dem Feinsediment liegt eine Schuttdecke aus groben, eingeregelten Kalkplatten, die aus hangaufwärts anstehenden, jungpliozänen Sedimenten stammen. Da Schuttdecken meistens unter periglazialen Bedingungen über Dauerfrostboden entstehen, kann sie, wie auch die liegenden Umlagerungszonen, aus quartärgeologischer Sicht dem ältesten Abschnitt der Würmeiszeit zugeordnet werden.

Über dem Solifluktionsschutt tritt ein $30 \mathrm{~cm}$ mächtiger, schwach feinsandiger, kalkhaltiger Schluff auf, der im unteren Bereich grünlichgelb und im oberen Abschnitt bräunlichgelb gefärbt ist. Seiner Ausbildung nach handelt es sich um typischen Löss, der jedoch im unteren Abschnitt aufgrund vereinzelt eingelagerter Kalkbröckchen schwache Anzeichen von Umlagerung besitzt. Auf diesem Löss hat sich die untere Mosbacher Humuszone (UMHZ) in einer Mächtigkeit von ca. $1 \mathrm{~m}$ entwickelt. Sie weist unterschiedlich ausgebildete Horizonte auf. So ist der untere Bereich als ein humusbrauner $\mathrm{B}_{\mathrm{V}}$-Horizont ausgebildet, in dessen oberem Abschnitt noch einzelne, unregelmäBig geformte Humusflecken erhalten sind. Die Degradation ist in diesem Horizont stark vorangeschritten. Im oberen Bereich der UMHZ ist dagegen als Grundsubstanz schwarzgraues Humuszonenmaterial vorhanden, welches nur vereinzelt von braunen Flecken durchsetzt ist (,Gefleckter Horizont" i. S. v. FINK 1954: 92 und Rohdenburg 1964: 66 ff.). Den Abschluss bildet ein graubräunlicher, schwach feinsandiger Schluff, bei dem es sich um umgelagertes Humuszonenmaterial handelt. 
Die gesamte UMHZ, insbesondere jedoch ihre verbraunten Abschnitte, weisen einen höheren Tongehalt auf als der liegende Löss, in dem sich ein $\mathrm{C}_{\mathrm{c}}$-Horizont gebildet hat (vgl. auch Abb. 2). $\mathrm{Da}$ der höhere Tongehalt vermutlich nicht auf Kryoklastik zurückzuführen ist, kann von einer chemischen Verwitterung in den Bodenhorizonten ausgegangen werden (vgl. BIBUs et al. 1996: 28, Semmel 1999a: 126). Von Bedeutung ist weiterhin die Tatsache, dass die UMHZ durch eine $8 \mathrm{~cm}$ mächtige Schicht aus hellgrauen Grobschluffbändern zweigeteilt ist. Die UMHZ ist somit polygenetisch und durch zwischenzeitliche Sedimentzufuhr gekennzeichnet.

Semmel (1999a: 126) hat für die Entstehung der UMHZ beispielhaft die Relief- und Bodenbildungsprozesse rekonstruiert. Danach soll es drei Schwarzerdebildungen, zwei Lösssedimentationsphasen mit dazwischenliegender Erosion, zwei Entkalkungsphasen und zwei Degradationsphasen gegeben haben. Im Prinzip können wir dieser Deutung folgen, wobei allerdings bei unseren Auf- nahmen in den tiefsten Bereichen der Delle der von Semmel beschriebene, basale Schwarzerdehorizont nicht vorhanden war. Dementsprechend können wir die UMHZ nur in zwei Bodenbildungsabschnitte (UMHZa = unterer Abschnitt der Humuszone mit starker Verbraunung bzw. Degradation und $\mathrm{UMHZb}=$ oberer Abschnitt der $\mathrm{Hu}-$ muszone mit geringer Degradation) untergliedern (vgl. Abb. 1).

Zwischen der unteren und mittleren Mosbacher Humuszone liegt ein $65 \mathrm{~cm}$ mächtiger Löss. Von Bedeutung ist, dass die untersten $50 \mathrm{~cm}$ dieses Lösses aufgrund der Anwesenheit von geringfügig aufgearbeitetem Humuszonenmaterial eine graustichige Farbe besitzen. Bei den Molluskenuntersuchungen ist deshalb damit zu rechnen, dass in diesem Abschnitt auch Arten der liegenden Humuszonen aufgearbeitet wurden.

Die mittlere Mosbacher Humuszone (MMHZ) ist wie die UMHZ nicht einheitlich (Abb. 1). Sie besitzt in ihrem unteren Bereich (MMHZa) einen

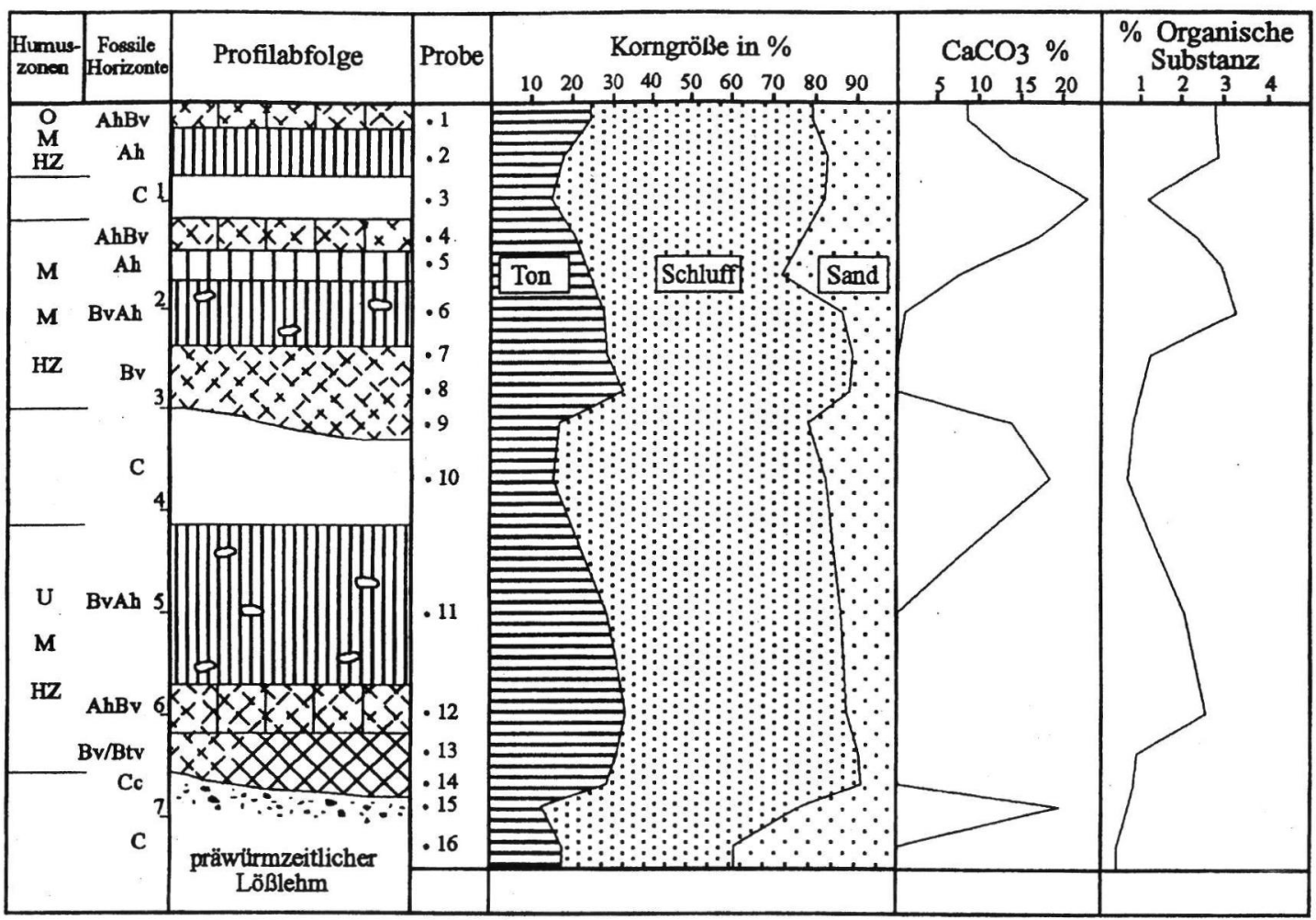

Abb. 2: Diagramm der Sedimentanalysen im Altwürmabschnitt der Delle 1 in Mainz-Weisenau (n. BiBus et al. 1996: 28).

Fig. 2: Diagram of sediment analysis of the Lower Wuermian section of depression number 1 in Mainz-Weisenau (according to BiBus et al. 1996: 28). 

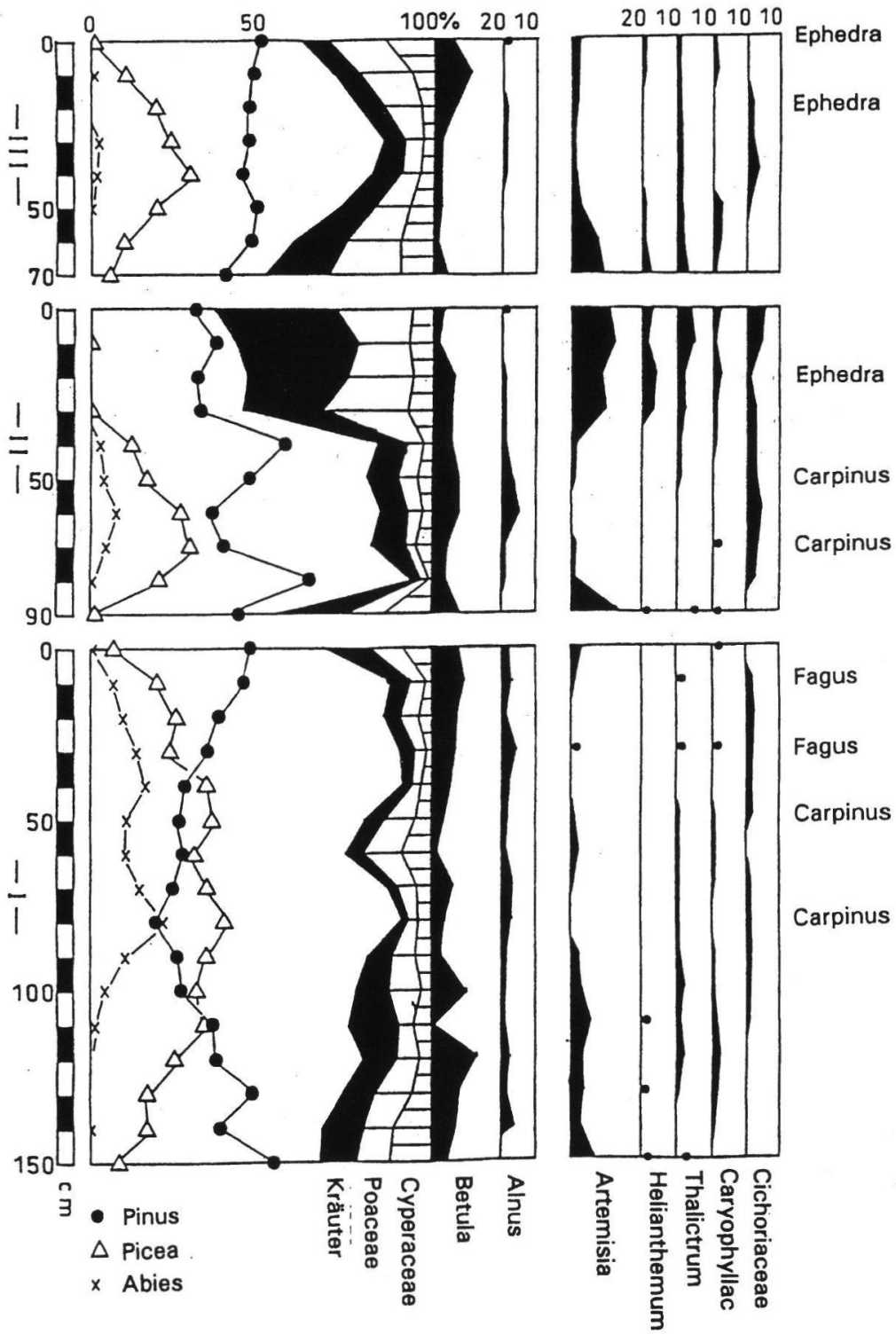

Abb. 3: Pollendiagramm der drei Altwürmhumuszonen in Delle 1 von Mainz-Weisenau (n. BLUdAu in Bibus et al. 1996). III = OMHZ, II = MMHZ, I = UMHZ.

Fig. 3: Pollen diagram of the three Lower Wuermian humus zones in depression number 1 in Mainz-Weisenau (according to BludAu in BiBus et al. 1996: 41). III = OMHZ, II = MMHZ, I = UMHZ.

$40 \mathrm{~cm}$ mächtigen $\mathrm{A}_{\mathrm{h}}$-Horizont mit einzelnen Verbraunungsflecken, die zur Untergrenze hin seltener werden. Im oberen Abschnitt (MMHZb) ist ein $35 \mathrm{~cm}$ starker $\mathrm{A}_{\mathrm{h}}$-Horizont ausgebildet. Zwischen diesen beiden Humushorizonten ist ein 30 $\mathrm{cm}$ mächtiger $\mathrm{B}_{\mathrm{v}}$-Horizont vorhanden. Es ist wahrscheinlich, dass sich auch hier, wie im Falle
Ephedra

der UMHZ, eine polygenetische Entstehung des gesamten Bodens abzeichnet. Nach SEMmeL (1999a: 125) soll im liegenden Löss der MMHZ ein $\mathrm{C}_{\mathrm{C}}$-Horizont entwickelt sein, der auf Entkalkungsvorgänge hinweist. Den Abschluss der MMHZ bildet ein schwarzbrauner, schwach lehmiger Schluff, der als umgelagertes Material der MMHZ gedeutet werden kann.

Der Löss zwischen der MMHZ und der oberen Mosbacher Humuszone (OMHZ) ist farblich zweigeteilt. Im oberen Abschnitt besitzt er die typische, gelblichgraue Lössfarbe, während der untere Abschnitt eine schwache Verbraunung aufweist. Möglicherweise ist der untere Bereich geringfügig verlagert.

Den Abschluss des Altwürmprofiles bildet die grauschwarze OMHZ mit einer Mächtigkeit von ca. $50 \mathrm{~cm}$. Im Gegensatz zu den beiden älteren Humuszonen ist sie ungegliedert und weist einen geringeren Anteil an organischer Substanz auf. Da sie sehr stark von Schnecken durchsetzt ist, muss von einer sehr geringen bzw. fehlenden Degradation ausgegangen werden. Dennoch weist auch sie einen um ca. $5 \%$ höheren Tongehalt gegenüber dem liegenden Löss auf (Abb. 2). Auch die jüngste Humuszone wird von umgelagertem Humuszonenmaterial überdeckt, welches lössstratigraphisch bereits in das mittlere Würm zu stellen ist. Nach SEMmel (1999a: 128) folgt im Hangenden eine mächtige, humose Schwemmlöss- 
decke, in der als typischer Leithorizont die Niedereschbacher Zone aus dem älteren Mittelwürmabschnitt ausgebildet ist.

An der Untergrenze der OMHZ war an einer Stelle ein Holzkohlehorizont mit einzelnen Knochensplittern und Rötelmaterial aufgeschlossen. Holzkohlenbestimmungen von $\mathrm{SCHOCH}$ (schriftl. Mitt.) haben ergeben, dass es sich um Nadelhölzer handelt, unter denen sich Pinus silvestris sowie Larix oder Picea befanden. Letztere konnten nicht genauer unterschieden werden. $\mathrm{Da}$ in dem von BLUDAU (in BIBUs et al. 1996) in einer angrenzenden Delle erstellten Pollendiagramm zwar Picea auftritt, aber Larix fehlt, kann davon ausgegangen werden, dass die nicht näher bestimmbaren Holzkohlen von Picea stammen (Abb. 3; vgl. auch SEMMEL 1995: 454). Obwohl bislang in der Holzkohlenlage keine Artefakte gefunden wurden, besteht die Möglichkeit, dass sie durch anthropogene, urgeschichtliche Eingriffe entstanden ist.

\section{Molluskenführung}

Was die Zusammensetzung der Molluskenfauna der OMHZ betrifft, so war bei der erneuten Beprobung eine weitgehende Übereinstimmung mit bereits früher (RAHLE in BIBUS et al. 1996) an einer anderen Stelle des Steinbruchs ermittelten Befunden festzustellen. Die Fauna der OMHZ setzt sich aus zwei ökologischen Komponenten zusammen, die auf eine enge Verzahnung unterschiedlicher Lebensräume hinweisen: erstens eine anspruchslose Waldsteppengemeinschaft (Fruticum-Fauna, mit Fruticicola fruticum, Orcula dolium, Vitrea crystallina und Arianta arbustorum), die aufgrund der lokalen Gegebenheiten (Dellenlage) einen mehr oder weniger hohen Anteil an ausgesprochen feuchtliebenden Schneckenarten wie Clausilia pumila und Perforatella bidentata aufweist, und zweitens die sog. Tridens-Fauna, die zahlenmäßig überwiegt und sich aus xerophilen Steppenschnecken wie Chondrula tridens, Granaria frumentum, Helicopsis striata und verschiedenen anderen Offenlandarten wie Pupilla muscorum, Vallonia costata, Vallonia pulchella und Vertigo pygmaea zusammensetzt. Dazu kommen klimaindifferente, mehr oder weniger ubiquitäre Formen wie kleine Nacktschnecken (Agriolimacidae), Cocblicopa lubrica, Vertigo substriata, Punctum pygmaeum, Nesovitrea bammonis und Trichia bispida.

Unterschiedliche Häufigkeiten einzelner Arten lassen sich auf zeitweilige oder lokale Unterschiede im Feuchtigkeitsangebot zurückführen. Während in der früher veröffentlichten Fauna (RÄHLE in BiBus et al. 1996: 26), die von einer zentralen Stelle der angrenzenden Delle 1 stammt, sowie in einer Molluskengesellschaft aus der in Kapitel 2 erwähnten Holzkohlen führenden Schicht an der Basis der OMHZ (Tab. 1, Proben 17 u. X) die Vertreter der Tridens-Fauna zahlenmäßig überwiegen, sind in Probe 18 die Dominanzverhältnisse deutlich zu Gunsten einer Fruticum-Fauna verschoben, in welcher feuchtliebende Arten (insbesondere Clausilia pumila und Perforatella bidentata) recht hohe Anteile erreichen.

Charakteristisch für Fruticum- und Tridens-Faunen ist das Fehlen von ausgesprochen warmzeitlichen oder kaltzeitlichen Elementen. Insgesamt lassen die Schnecken auf ein mäßig kühles, sommerwarmes Kontinentalklima schließen (LOžEK 1964).

Weit weniger klar und eindeutig erwiesen sich die Verhältnisse im Liegenden der OMHZ. In nahezu allen Horizonten waren Reste von warmzeitlichen Arten festzustellen, nicht nur in der MMHZ und UMHZ, sondern auch in den Sedimenten dazwischen und selbst in den als Löss angesehenen Ablagerungen. Es ist also an verschiedenen Stellen des Profils mit einem nicht unerheblichen Anteil von umgelagertem Schalenmaterial zu rechnen. Dieser Befund ist angesichts der Hanglage der Fundstelle, der komplizierten Genese des gesamten Humuszonenkomplexes und der an vielen Stellen auch sedimentologisch direkt nachweisbaren Sedimentverlagerungen (siehe Profilbeschreibung) nicht überraschend und muss bei der Faunenanalyse berücksichtigt werden.

Der $\mathrm{fB}_{\mathrm{t}}$-Horizont und die darüberliegende Fließerde aus $\mathrm{B}_{\mathrm{t}}$-Material (Tab. 1, Probe 1) haben eine Fauna mit einigen wenigen Arten geliefert, welche für Interglaziale charakteristisch sind (Carychium tridentatum, Discus rotundatus, Limax spec.). Sie kann als Fragment einer späteemzeitlichen Fauna angesehen werden. Fast zwei Drittel der Individuen entfallen auf die Offenlandarten (Truncatellina cylindrica, Pupilla muscorum). Zu diesen zählt auch Cecilioides acicula, eine weitere warmzeitlichen Schnecke, auf deren Vorkommen weiter unten eingegangen wird. Insgesamt lassen die Funde auf eine weitgehend offene Landschaft mit spärlicher höherer Vegetation schließen.

Bezeichnend für die Horizonte aus feinsandigem Schluff, Solifluktionsschutt und Löss zwischen dem $\mathrm{fB}_{\mathrm{t}}$-Horizont und der UMHZ (Tab. 1, Pro- 
ben 2 - 5) ist das relativ zahlreiche Auftreten hochglazialer Indexarten wie Columella columella, Vertigo parcedentata, Vallonia tenuilabris und Pupilla loessica. Dieser Befund scheint zunächst für eine extreme Absenkung der Temperaturen im Anschluss an die letzte Warmzeit zu sprechen und steht voll im Einklang mit den in diesem Profilabschnitt nachgewiesenen periglazialen Erscheinungen wie Solifluktion und Lössbildung. Daneben finden sich überraschenderweise aber auch Reste einer ganzen Anzahl von warmzeitlichen Spezies wie $C a$ rychium tridentatum, Sphyradium doliolum, Ena montana, Macrogastra spec., Ruthenica filograna, Discus rotundatus, Aegopinella spec., Limax spec., Helicodonta obvoluta, Isognomostoma isognomostomos und Cepaea spec.. Dazu kommen Arten wie Vertigo pygmaea, Vallonia enniensis, Fruticicola fruticum und Euomphalia strigella, die vorwiegend warmzeitlich sind und nur in milderen Abschnitten von Kaltzeiten auftreten.

Die ökologischen Ansprüche der vorkommenden Arten sind so unterschiedlich, dass die Annahme, es könne sich hier um autochthone Molluskenbestände handeln, auszuschließen ist. Vielmehr lässt sich die Molluskenfauna aus diesem Profilabschnitt als Mischfauna mit autochthonen und allochthonen Elementen beschreiben, welche nicht ohne weiteres voneinander zu trennen sind.

Verantwortlich für eine solche Durchmischung dürfte eine kräftige Erosions- und Umlagerungsphase sein, die nach der Bildung der Parabraunerde einsetzte (SEMMEL 1999a), und die nicht nur den $\mathrm{A}_{\mathrm{l}}$ - und einen Großteil des Bt-Horizontes entfernt, sondern möglicherweise sogar die älteren Liegendschichten erfasst hat, so dass es zu einer Verlagerung von unterschiedlich altem Material in das Zentrum der Delle gekommen ist. Wie die Molluskenfunde zeigen, müssen solche Umlagerungsprozesse auch noch zur Zeit der Lössbildung angedauert haben (Tab. 1, Probe 5).

Als sekundär eingemischt können aus sedimentologischen Gründen sehr wahrscheinlich alle warmzeitlichen Arten angesehen werden, die in diesem Profilabschnitt vorkommen. $\mathrm{Ob}$ es sich dabei lediglich um umgelagerte Eem-Mollusken handelt, oder ob sie möglicherweise aus noch älteren Ablagerungen stammen, ist schwer zu sagen. Bei kritischer Würdigung der gesamten Fundlage ist dagegen die Annahme einer sekundären Einmischung auch der kaltzeitlichen Faunenelemente eher unwahrscheinlich. Unseres Erachtens kann man davon ausgehen, dass diese Faunenkomponente den Einzug eines verhältnismäßig feuchten, subarktischen Klimas am Beginn der Würmeiszeit widerspiegelt.

Bei den kaltzeitlichen Indexarten sind die Funde von Vallonia tenuilabris besonders bemerkenswert. Es handelt sich um eine zentralasiatisch-sibirische Art, welche am Oberrhein und weiter westlich bis vor wenigen Jahren noch als Leitart für präeemzeitliche Glaziale angesehen wurde (u.a. MüNZING 1985, RousSEAU 1986). Inzwischen liegen aber auch aus diesem Gebiet Nachweise aus würmzeitlichen Fundzusammenhängen vor (MÜNZING \& LÖsCHER 1985). Auch im Würmlöss von Koblenz-Metternich konnte Vallonia tenuilabris nachgewiesen werden (RÄHLE unveröff.). Ein Vorkommen dieser Art im Rhein-Main-Gebiet und benachbarten Regionen in der Würmeiszeit dürfte also als gesichert gelten.

In der UMHZ wurden keinerlei kaltzeitliche Faunenelemente festgestellt. Im unteren, stark degradierten Abschnitt (UMHZa, Abb. 1) waren nur ganz wenige Mollusken zu finden, die keine klimaökologischen Aussagen zulassen (Tab. 1, Probe 6). Reichlicher vertreten waren die Mollusken im oberen, nicht verbraunten Abschnitt der UM$\mathrm{HZb}$ (Tab. 1, Probe 7). Bemerkenswert ist die groBe Zahl der dort nachgewiesenen Arten. Neben Offenlandbewohnern (Helicopsis striata, Cecilioides acicula, Pupillen und Vallonien) und einigen anspruchslosen Ubiquisten (Agriolimacidae, Succinella oblonga, Trichia hispida) führt dieser Horizont zahlreiche Reste von ausgesprochenen Waldarten (Sphyradium doliolum, Cocblodina laminata, Macrogastra ventricosa?, Macrogastra plicatula, Ruthenica filograna, Aegopinella spec., Monachoides incarnatus, Helicodonta obvoluta). Dazu kommen einige wärmeliebende, mesophile Arten wie Discus rotundatus, Helicigona lapicida und Cepaea spec., die eine deutliche Präferenz für Waldbiotope zeigen. Ungefähr ein Drittel der Arten und ca. $20 \%$ der Individuen entfällt auf diese biostratigraphisch bedeutsame Gruppe. Auch Euomphalia strigella und Fruticicola fruticum, typische Arten der Waldsteppe, sind nachzuweisen. Die Arten der offenen Landschaft sind dagegen deutlich schwächer vertreten als in den anderen Horizonten des Profils.

Auch in der darüberliegenden, feinsandig-schluffigen Zone aus umgelagertem Humuszonenmaterial und in dem schmutzig-grauen, schwach humosen Löss über dieser Umlagerungszone (Tab. 1, Proben 8 und 9) wurden vereinzelt Schalenres- 
9

$x$

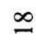

ב

$\underline{0}$

$\because$

$\pm$

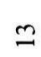

$\simeq$

$=$

으

$a$

$\infty$

r

○

in

$+$

m

N

$-$

$\circ$

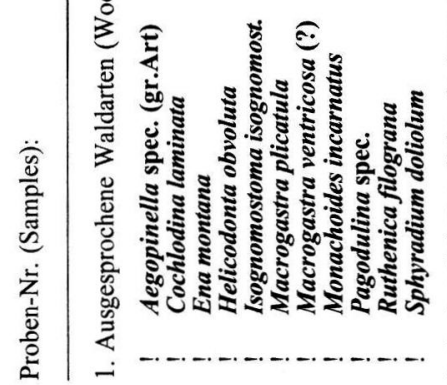

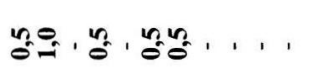

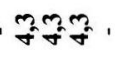

$\hat{\theta}, \hat{\theta} \hat{\theta} \cdot \hat{\theta} \hat{\theta}$

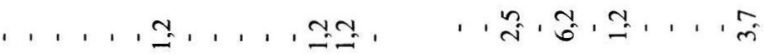

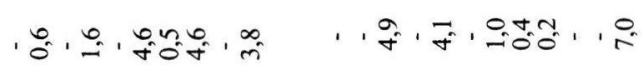

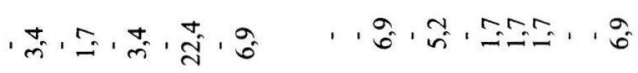

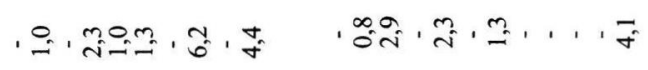

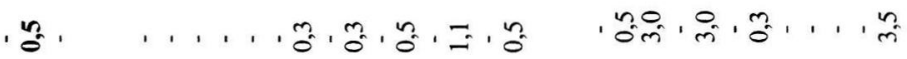

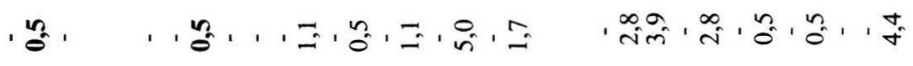

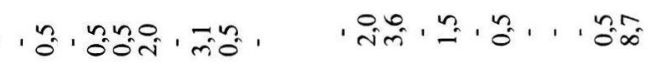

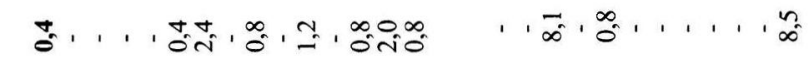

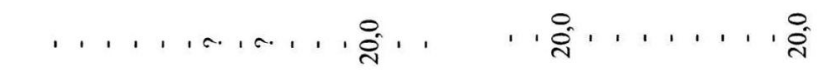

이.

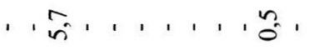

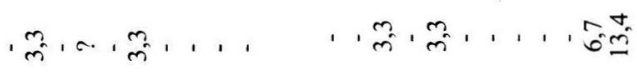

$\hat{\infty}+1, \ldots$

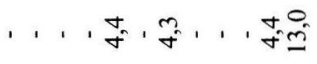

, t.

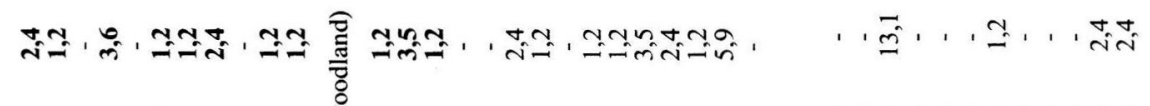

, 苑, , , 灾.

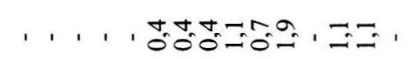

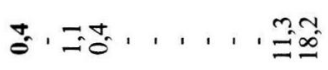

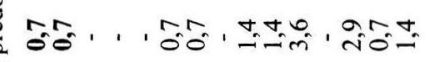

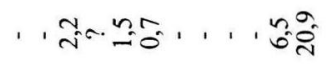
के

. मimon. है

a. 2.

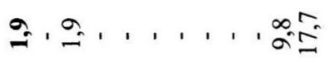

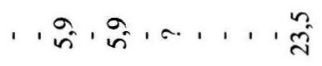

in. .
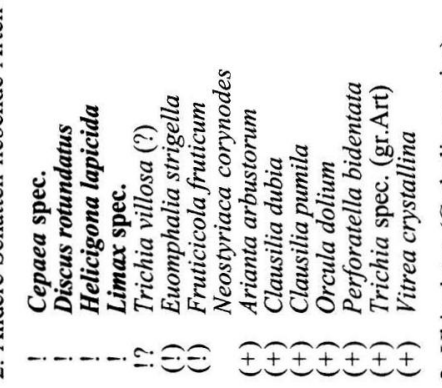
4. Offenlandarten (Open-country species)

$\begin{array}{ll}\text { !) } & \text { Cecilioides acicula } \\ (!) & \text { Truncatellina cylindrica } \\ (!) & \text { Vallonia enniensis } \\ (!) & \text { Vallonia excentrica } \\ (!) & \text { Vallonia pulchella } \\ (!) & \text { Vertigo pygmaea } \\ (+) & \text { Chondrula tridens } \\ (+) & \text { Granaria frumentum } \\ (+) & \text { Pupilla bigranataltriplicata } \\ (+) & \text { Vallonia costata } \\ + & \text { Helicopsis striata } \\ + & \text { Pupilla muscorum } \\ + & \text { Pupilla sterrii } \\ ++ & \text { Columella columella } \\ ++ & \text { Pupilla loessica } \\ ++ & \text { Vallonia tenuilabris } \\ ++ & \text { Vertigo parcedentata }\end{array}$

$\begin{array}{cc}- & \mathbf{7 , 9} \\ - & 4,0 \\ - & - \\ - & - \\ - & - \\ - & - \\ - & - \\ - & - \\ - & - \\ 17,6 & - \\ 5,9 & - \\ 35,3 & 51,0 \\ - & - \\ - & - \\ - & - \\ - & - \\ - & -\end{array}$

8

$\begin{array}{ccc}10 & 11 & 12 \\ & & \\ - & - & - \\ - & - & - \\ - & - & - \\ - & 0,5 & - \\ 6,7 & - & - \\ 3,3 & 4,2 & - \\ 3,3 & 9,3 & - \\ - & - & - \\ - & 1,0 & - \\ 26,7 & 48,8 & - \\ 3,3 & 17,1 & 20,0 \\ 23,4 & 8,3 & - \\ - & - & - \\ - & - & - \\ - & - & - \\ - & - & - \\ - & - & -\end{array}$

$\begin{array}{lllllllll}13 & 14 & 15 & 16 & 17 & 18 & \mathrm{X} & 19\end{array}$

5. Wassermollusken (Aquatic molluscs)
(!) Ancylus fluviatilis
(!) Bithynia tentaculato
(!) Bythiospeum spec.
(!) Bythiospeum spec.
(+) Radix peregra
(+) Valvata spec.

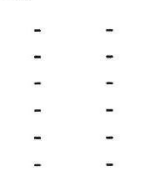

Unbestimmte Landschnecken (not clearly identified landsnails)

\begin{tabular}{|c|c|c|c|c|c|c|c|c|c|c|c|c|c|c|c|c|c|c|c|c|c|}
\hline Clausiliidae (indet.) & - & 2,0 & 5,3 & 4,1 & 1,5 & 1,5 & 20,0 & 3,5 & 3,0 & 4,4 & - & 2,1 & - & 0,4 & - & - & - & - & - & - & - \\
\hline Trichia spec. & - & - & - & - & - & - & - & $\therefore$ & 6,1 & - & - & - & - & - & - & - & - & - & - & - & - \\
\hline Vallonia spec. & - & - & & - & 2,2 & & 20,0 & & & - & - & - & - & - & - & - & - & - & - & - & - \\
\hline ahl (Number o & 7 & 10 & 22 & 27 & 29 & 25 & 4 & 31 & 13 & 14 & 12 & 14 & 5 & 20 & 26 & 20 & 18 & 21 & 14 & 19 & 14 \\
\hline iduen (Individuals) (= $100 \%)$ : & 17 & 51 & 112 & 296 & 139 & 264 & 5 & 84 & 33 & 23 & 30 & 193 & 5 & 247 & 197 & 182 & 369 & 384 & 58 & 184 & 81 \\
\hline
\end{tabular}

Tabelle 1: Molluskenfaunen aus dem Altwürmabschnitt von Mainz-Weisenau. Die Entnahmestellen der Proben 0 - 19 (Delle 2 ) sind der Abb. 1 zu entnehmen; X = Fauna aus der OMHZ in Delle 1 (n. Bibus et al. 1996: 26), stammt nicht aus dem in Abb. 1 wiedergegebenen Profil. Angegeben sind die Dominanzwerte (= Anteile der Arten an der Gesamtindividuenzahl einer Probe) in \%. Biostratigraphische Charakteristik in Anlehnung an LožEK (1964): ! - warmzeitliche Arten, (!) - vorwiegend warmzeitliche Arten (auch in milderen Abschnitten der Kaltzeiten), (+) - klimatisch indifferente Arten, + - klimatisch indifferente Arten (in Kaltzeiten besonders häufig), ++ - hochkaltzeitliche Indexarten. Biostratigraphisch besonders bedeutsame Arten sind durch Fettdruck hervorgehoben.

Table 1: Mollusc faunas of the Lower Wuermian section in Mainz-Weisenau. The origin of the samples 0 to 19 (depression 2) can be seen in fig. 1; X = fauna from the Upper humic zone of depression 1 (according to BiBus et al. 1996: 26). The frequency of species per sample is expressed by dominance rates in \%. ! - species characteristic of warm phases, (!) - mainly in warm phases, but also in warmer periods of glacials, (+) - climatically indifferent species, + - climatically indifferent species, but particularly common in glacials, ++ - index species of glacials. Biostratigraphically important species are set in bold. 
te thermisch anspruchsvoller Arten festgestellt. Dass es sich dabei um umgelagerte Schalen handelt, die aus der UMHZ stammen, ist sehr wahrscheinlich.

Alle Funde zusammengenommen - sowohl aus der UMHZ selbst als auch aus dem umgelagerten Humuszonenmaterial darüber - bezeugen das zeitweise Vorhandensein von Gehölzbeständen während der Bildung der UMHZ und ein gemäBigt warmes Klima, wenigstens in der zweiten Hälfte dieses Zeitabschnittes. Dabei ist festzuhalten, dass alle in diesem Bereich nachgewiesenen Waldarten auch in Nadelwaldbeständen leben können, und nicht auf Laubgehölze angewiesen sind. Die malakologischen Befunde stehen damit voll im Einklang mit den Ergebnissen der von BLUDAU durchgeführten Pollenanalyse („,Kiefern-Fichten-Tannenzeit", BLudAu in BiBus et al. 1996; vgl. Abb. 3).

Der Löss zwischen UMHZ und MMHZ ist nur in seinem obersten Teil, einem $15 \mathrm{~cm}$ mächtigen Band aus hellem Löss, welches unter der MMHZ liegt (Tab. 1, Probe 10), frei von Beimengungen warmzeitlicher Molluskenarten. Es kommen aber auch keine ausgesprochen kaltzeitlichen Faunenelemente vor. Es gibt also keine Hinweise auf eine gravierende Absenkung der Temperaturen zur Zeit dieser Lössbildung. Das Artenspektrum setzt sich hier lediglich aus Angehörigen der Fruticum-Fauna und ihrer Steppenanalogie, der Tridens-Fauna, zusammen, was auf eine mäßig kühle Steppe und lokal auch Waldsteppe schließen lässt.

Die MMHZ ist mehrgliedrig. Im Gegensatz zu ihrem unteren und oberen Abschnitt (MMHZa bzw. MMHZb) waren in dem $\mathrm{B}_{\mathrm{v}}$-Horizont dazwischen, wohl infolge stärkerer Entkalkung, nur ganz wenige Fossilien zu finden. Sie lassen keine Schlussfolgerungen klimaökologischer Art zu und werden deshalb bei der Faunenanalyse nicht weiter berücksichtigt (Tab. 1, Probe 12). In der MMHZa (Tab. 1, Probe 11) und der MMHZb (Tab. 1, Probe 13) treten in Faunen, die ganz geprägt sind von Elementen der Tridens- und Fruticum-Fauna, spärliche Reste von warmzeitlichen Waldarten auf (Cochlodina laminata, Monachoides incarnatus bzw. Cepaea spec.).

Auf die MMHZ folgen, wie im Hangenden der UMHZ, umgelagertes Humuszonenmaterial und Löss, der in seinem unteren Teil ebenfalls verlagertes Humuszonenmaterial enthält. In beiden
Schichten treten Reste von weiteren warmzeitlichen Arten auf wie Macrogastra ventricosa (?), Macrogastra plicatula, Ruthenica filograna, Aegopinella spec., Helicodonta obvoluta und Helicigona lapicida. Die Mehrzahl derartiger Fossilien hat eine undifferenziert entnommene Mischprobe geliefert (Tab. 1, Probe 14). Es handelt sich dabei ausnahmslos um Formen, die auch im Bereich der UMHZ gefunden wurden. Etliche davon sind ausgesprochene Waldarten. Dass diese biostratigraphisch bedeutsamen Arten in dem verlagerten Humuszonenmaterial reichlicher vertreten sind als in der Humuszone selbst, könnte mit der Größe des Einzugsbereiches zusammenhängen, der von den Verlagerungsprozessen erfasst wurde.

$\mathrm{Ob}$ und in welchem Ausmaß von den Erosionsund Verlagerungsvorgängen auch Sedimente im Liegenden der MMHZ erfasst wurden, lässt sich schwer sagen. Da nach den Ausführungen von SeMmel (1999a) die MMHZ an manchen Stellen die UMHZ schneidet, muss mit der Möglichkeit und damit auch mit der Einmischung älteren Fossilmaterials gerechnet werden. Vorerst wird jedoch davon ausgegangen, dass - analog zu den Verhältnissen über der UMHZ - der weitaus überwiegende Teil der hier nachweisbaren warmzeitlichen Elemente aus Material der MMHZ stammt. Im Gegensatz zur UMHZ treten die im Bereich der MMHZ gefundenen, klimatisch besonders anspruchsvollen Elemente zahlenmäßig ganz in den Hintergrund (5 - 20\% der Arten, 1 - $3 \%$ der Individuen). Die Fauna wird hier in ungleich stärkerem Maße von Offenlandarten, darunter typische Steppenarten wie Chondrula tridens und Helicopsis striata, dominiert.

Folgt man der vorgenommenen Interpretation des vorliegenden Fossilberichts, dann ergibt sich, dass die MMHZ ein Interstadial repräsentiert, dessen Intensität etwas geringer war als diejenige des vorhergehenden. Eine solche Deutung passt gut zu den palynologischen Befunden [,Kiefern-Fichten (Tannen)-Zeit“ bzw. „Kiefern-Zeit“ bei BLudau in Bibus et al. 1996; vgl. Abb. 3].

Von besonderem Interesse ist eine in allen $\mathrm{Ab}-$ schnitten der MMHZ vorkommende, nicht näher zu identifizierende Art der Gattung Bythiospeum. Sie tritt auch in der UMHZ (Tab. 1, Probe 7) und in der OMHZ auf (Tab. 1, Probe 17). Den Lebensraum von Bytbiospeum bilden das Grundwasser und andere unterirdische Spaltengewässer. Es müssen also in nicht allzu großer Entfernung 
Quellen vorhanden gewesen sein, in denen die Schalen ausgeworfen worden sind. Es ist möglich, dass solche Quellaustritte für ein erhöhtes Feuchtigkeitsangebot gesorgt haben, was die Ausbildung höherer Vegetation günstig beeinflusst hat.

Im Löss zwischen MMHZ und OMHZ wurde zwei Mal Vallonia tenuilabris nachgewiesen. Der eine der vorliegenden Funde stammt aus dem Standardprofil (Tab. 1, Probe 16), der andere aus einer undifferenziert entnommenen Probe, die offensichtlich auch verlagertes Humuszonenmaterial enthielt (Tab. 1, Probe 14). Vallonia tenuilabris bewohnt heute in Zentralasien und Sibirien verschiedene Lebensräume wie karge Steppen und Gebirgssteppen, den Taigagürtel und die Tundren-Region (Gerber 1996). In Europa tritt sie ausschließlich in pleistozänen Kaltzeiten auf. Hält man die Vorkommen von Vallonia tenuilabris im Löss zwischen MMHZ und OMHZ für autochthon, dann wäre das als Hinweis auf eine bedeutende Absenkung der Durchschnittstemperaturen und für den Einzug eines extrem kontinentalen Klimas nach der Bildung der MMHZ zu werten.

Im Bereich der Mosbacher Humuszonen tritt in Mainz-Weisenau in zahlreichen Horizonten die Blindschnecke Cecilioides acicula auf ( $\mathrm{fB}_{\mathrm{t}}$-Horizont, Löss im Liegenden der UMHZ, UMHZ samt umgelagertem Humuszonenmaterial darüber, Löss unter der $\mathrm{OMHZ}$ ). In Hangendschichten der OMHZ und in der OMHZ selbst ist die Schnecke dagegen nicht mehr nachzuweisen. Es handelt sich um eine wärmeliebende, süd- und mitteleuropäische Art. Sie lebt subterran in lockeren Böden an trockenen, vorwiegend offenen Standorten. Dabei dringt sie entlang von Regenwurmröhren und Pflanzenwurzeln bis in eine Tiefe von ca. zwei Metern vor (Evans 1972: 80). Leere Schalen werden oft durch wühlende Kleinsäuger an die Oberfläche befördert. Vorkommen von Cecilioides acicu$l a$ in jungpleistozänen Schichten werden in der Regel als holozäne Einmischungen betrachtet (LOŽEK 1964, Mania 1973: 79). Im Falle von MainzWeisenau, wo die Fundhorizonte unter mehr als 5 Meter mächtigen Deckschichten aus Würmlöss liegen, ist eine solche Deutung auszuschließen. Die Funde lassen sich hier eindeutig dem $\mathrm{fB}_{\mathrm{t}}$-Horizont, der UMHZ und auch noch der MMHZ zuordnen. Sie sind also biostratigraphisch durchaus verwertbar und ein weiteres Indiz für die thermisch günstigen Verhältnisse in diesen beiden Interstadialen.

\section{Zusammenfassende Diskussion}

In der Abfolge von Mainz-Weisenau lässt der Fossilbericht, trotz Störungen durch Umlagerungsvorgänge nach dem letzten Interglazial, drei Wärmeschwankungen erkennen, die mit den Humuszonen korrelierbar sind und in ihrer Intensität von unten nach oben abnehmen. Zwar dominieren fast überall die Offenlandarten, doch liegen aus dem Bereich der UMHZ und MMHZ Faunen vor, die für gemäßigte Klimaverhältnisse und für die lokale Existenz von Wäldern sprechen, wenigstens zu Zeiten des Optimums der betreffenden Interstadiale. Das gilt insbesondere für die UMHZ, wo Waldarten besonders zahlreich anzutreffen waren. Für die OMHZ ist dagegen nur noch Steppe und wohl lokal, an mikroklimatisch besonders begünstigten Stellen, auch Waldsteppe mit mäßig kühlem, kontinentalem Klima belegt.

Auf Grund der Lage der Fundstelle in einer Delle muss mit ganz besonderen ökologischen Bedingungen gerechnet werden. Die windgeschützte Lage und ein erhöhtes Feuchtigkeitsangebot, welches sich auch in den Molluskenfaunen widerspiegelt, dürfte nicht ohne Auswirkungen auf die Vegetation geblieben sein, so dass ein unmittelbarer Vergleich mit Ergebnissen entsprechender Untersuchungen an anderer Stelle nur bedingt möglich ist. Letzteres gilt insbesondere für den Vergleich mit den Ausführungen bei REMY $(1968,1969)$, der die Molluskenführung der Mosbacher Humuszonen im Gebiet des Rheingaues und Rheinhessens genauer untersucht hat.

In Mainz-Weisenau treten in der UMHZ und MMHZ keine waldgebundenen Schneckenarten auf, die nur unter Laubwald vorkommen. Die nachgewiesenen Arten können alle auch in Nadelgehölzen existieren. Dieser Befund deckt sich mit den Ergebnissen der palynologischen Untersuchungen (BludAu in BiBus et al. 1996), nach denen es sich bei den Gehölzen überwiegend um Nadelbäume (Pinus, Picea, Abies) gehandelt hat (vgl. Abb. 3). Wie bei den Mollusken nehmen auch in der Vegetation die klimatischen Ansprüche in den Humuszonen von der unteren nach der oberen ab.

REMY hat im Dyckerhoff - Steinbruch bei Wiesbaden sowie in Wallertheim (Ziegeleigrube Schick im Wiesbachtal) alle drei Mosbacher Humuszonen beprobt und konnte lediglich wärmere oder kühlere Steppenfaunen vom Typ der Tridens- oder 
Striata-Fauna i. S. v. LožEK (1964) nachweisen (REMY 1968: 129 und 131, Tab. 3, A-C, Tab. 4, DF; RemY 1969: 106 - 107 und 109, Tab. 3 und Tab. 4, I-III). Warmzeitliche Arten, wie sie jetzt aus der UMHZ und MMHZ von Mainz-Weisenau vorliegen, hat Remy nicht gefunden. Auch hat RemY an keiner der von ihm im Rheingau und Rheinhessen untersuchten, altwürmzeitlichen Fundstellen Faunen mit hochglazialen Leitarten nachweisen können. Er erwähnt indessen altwürmzeitliche Molluskengesellschaften mit Columella columella aus dem mittelrheinischen Gebiet (REMY 1968: 140; 1969:111).

Die Abfolge der Molluskenfaunen aus dem unteren Teil des Profils Mainz-Weisenau weist bemerkenswerterweise viele Gemeinsamkeiten mit Faunen auf, welche von MANIA $(1978,1997)$ aus den Deckschichten der eemzeitlichen Travertine von Burgtonna in Thüringen und aus dem Lössprofil von Wallertheim in Rheinhessen beschrieben worden sind. Auch dort ist in weitgehend von Offenlandarten beherrschten Waldsteppengesell-schaften ein zweimaliges Auftauchen von Arten belegt, die sonst nur aus Interglazialen bekannt sind und auf lokale Bewaldung und ein gemäßigtes Klima hinweisen. Auffällig ist dabei, dass es sich annähernd um die selben, verhältnismäßig genügsamen Arten handelt, die in Mainz-Weisenau in der UMHZ und MMHZ auftauchen (Discus rotundatus, Cochlodina laminata, Ruthenica filograna, Monachoides incarnatus, Helicodonta obvoluta, Helicigona lapicida und Cepaea spec.). Da an beiden Fundstellen in den Zwischenschichten keinerlei Anzeichen für kaltzeitliche Klimaverhältnisse festgestellt werden konnten, kommt für MaNIA (1997) eine Einstufung der von ihm bearbeiteten Faunen in altwürmzeitliche Interstadiale nicht in Frage. Sie werden vielmehr als Faunen aus wärmeren Schwankungen in einer kontinental geprägten Spätphase des letzten Interglazials interpretiert. Hinweise auf instabiles Klima in der zweiten Hälfte des Eem und periodisch wiederkehrende Kälteeinbrüche sind in letzter Zeit von verschiedener Seite erbracht worden (DANSGAARD et al. 1993; FiELD et al. 1994; Cortijo et al. 1994).

Auf größere Schwierigkeiten stößt man in MainzWeisenau, wenn es um die biostratigraphische Interpretation der Faunen aus dem Liegenden der Humuszonen geht. Pollenanalytische Untersuchungen, die zum Vergleich herangezogen werden könnten, liegen z. Zt. nicht vor. Während im Löss, unmittelbar unter der MMHZ, eine Fauna vorge- funden wurde, die ihrer Zusammensetzung nach sehr an diejenige aus der OMHZ erinnert und relativ kühles aber nicht kaltes, sommerwarmes Kontinentalklima bezeugt, treten vor allem im Liegenden der UMHZ, aber auch der OMHZ, hochkaltzeitliche Arten auf. Solche Anzeiger einer extremen Absenkung der Durchschnittstemperaturen kommen in Mainz-Weisenau in Mischfaunen vor, und es besteht, wie bereits oben dargelegt, keine letzte Sicherheit über deren autochthone Vorkommen. Durch die Anwesenheit kaltzeitlicher Leitarten unterscheidet sich die Faunenabfolge in Mainz-Weisenau deutlich von denjenigen, die von MANiA in Burgtonna oder Wallertheim angetroffen wurden. Ob und inwieweit die Befunde in Wallertheim für einen Vergleich mit den Verhältnissen in Mainz-Weisenau in Frage kommen, ist wegen der Lückenhaftigkeit des Wallertheimer Profils allerdings sehr problematisch (vgl. SEMMEI 1999a: 123).

Bei Annahme eines autochthonen Vorkommens der kaltzeitlichen Molluskenarten ließe sich der vorliegende Fossilbericht indessen mit den allgemein akzeptierten Vorstellungen über die Klimaentwicklung am Beginn des Würmglazials, wie sie sich in den Tiefsee- und Eisisotopenkurven abzeichnet, recht gut vereinbaren. Unabhängig von der Molluskenführung belegen die periglazialen Solifluktionsphasen zwischen dem letztinterglazialen Boden und der UMHZ ein frühestwürmzeitliches Alter. Es kann deshalb gefolgert werden, dass es sich bei den aus der UMHZ und MMHZ vorliegenden anspruchsvollen Faunen, die für gemäßigte Klimaverhältnisse und einen gewissen Grad an Wiederbewaldung sprechen, tatsächlich um altwürminterstadiale und nicht um späteemzeitliche Faunen und Böden handelt.

Zudem kann die UMHZ mit dem eemzeitlichen $\mathrm{B}_{\mathrm{t}}$-Horizont in keine genetische Beziehung gebracht werden, da der A1-Horizont der Parabraunerde durch eine zwischengeschaltete Erosionsphase abgetragen wurde und mehrgliedrige Sedimente (Löss, Solifluktionsdecken) zwischen den beiden Bodenhorizonten auftreten. Eine Parallelisierung der UMHZ und der MMHZ mit den norddeutschen bewaldeten Interstadialen Amersfoort/ Brörup und Odderade halten wir weiterhin für wahrscheinlich (vgl. BiBus et al. 1996), zumal die Molluskenuntersuchungen in Übereinstimmung zu den Pollenanalysen stehen. Der Abschnitt vom 1. $\mathrm{fB}_{\mathrm{t}}$ bis zur MMHZ kann somit in das Tiefseeisotopenstadium 5 gestellt werden. Möglicherwei- 
se spiegelt dabei die Zweigliederung der UMHZ die Zweigipfligkeit des Isotopenstadiums $5 c$ wider. Für die Zeit zwischen der Bildung der MMHZ und der OMHZ gibt es bei den Mollusken Hinweise auf eine Phase mit extrem kaltem Klima, welche den Beginn des Mittelwürms (marines Isotopenstadium 4) markieren könnte. Die OMHZ, welche nach den vorliegenden Molluskenfunden ein thermisch deutlich weniger günstiges Interstadial repräsentiert als die MMHZ und UMHZ, wäre dann mit dem frühen mittelwürmzeitlichen Interstadial des Oerel (ältester Abschnitt des marinen Isotopenstadiums 3) zu korrelieren.

Absolute Datierungen, die im Löss zwischen MMHZ und OMHZ durchgeführt wurden (FRECHEN \& Preusser 1996) und Alter zwischen 72,4 $\pm 12,3 \mathrm{ka}$ (IRSL/ADD) und 77,6 $\pm 18,9 \mathrm{ka}(\mathrm{TL} /$ ADD) ergeben haben (vgl. auch ZöLLER, zit. bei Semmel 1996a: 13), stehen zumindest nicht im Widerspruch zu einer solchen Zuordnung. Andererseits ist es nicht auszuschließen, dass die OMHZ noch in den Abschnitt 5a gehört, der nach der ${ }^{18} \mathrm{O}$-Kurve im grönländischen Eis in sich noch weiter untergliedert ist (GRIP 1993, DANSGAARD et al. 1993). So würde sich eine Korrelation mit dem zweigipfligen Interstadial Nr. 19 und 20 anbieten. Gegen eine Parallelisierung des Oerel-Interstadials mit der OMHZ spricht nach SEMMEL (1999a) die Tatsache, dass das Oerel-Interstadial in Norddeutschland nicht bewaldet war, in dem Pollendiagramm der OMHZ jedoch eine Nadelwaldvegetation nachgewiesen wurde (Abb. 3). Auch in den Molluskenfaunen (Tab. 1, Proben 17, 18 und $\mathrm{X}$ ) kommen, neben vielen Offenlandbewohnern, einige Arten vor, die auf höhere Vegetation angewiesen sind. Wahrscheinlich muss im Altwürm ein stärkerer Temperaturgradient zwischen Nord- und Süddeutschland berücksichtigt werden, wie dies von BEHRE \& LADE (1986) sowie CASPERS \& FREUND (1998) vertreten wird. Neuere Pollenanalysen im ehemaligen Rheingletschergebiet (MüLLER 2000) haben zumindest gezeigt, dass eine pollenanalytische Korrelation zwischen den St. Germain-Interstadialen und den beiden ältesten süddeutschen Altwürminterstadialen und diese wiederum mit den norddeutschen älteren Weichselinterstadialen möglich ist.

Im Gegensatz zu früheren Ansichten halten wir deshalb neuerdings eine Parallelisierung von St. Germain I und St. Germain II mit Brörup und Odderade für wahrscheinlich. Es wäre auch sicherlich nicht erklärlich, dass in den jeweils kom- plettesten Profilen der verschiedenen Regionen über dem Eem jeweils verschiedenen alte Interstadiale überliefert sind.

Abschließend sei noch darauf hingewiesen, dass von ANTOINE (1998) und ANTOINE et al. (1998) in Nordwestfrankreich im Altwürm vor der Bildung der Steppenböden ein grauer Waldboden nachgewiesen wurde, der zwischen $67 \mathrm{ka}$ und $107 \mathrm{ka}$ entstanden sein soll. $\mathrm{Da}$ ein solcher Boden bislang in Mitteleuropa unter den drei Humuszonen noch nicht nachgewiesen wurde, muss offen bleiben, $\mathrm{ob}$ dieser graue Waldboden ein Äquivalent der UMHZ (möglicherweise auch der UMHZ und der MMHZ) ist, oder ob es sich um eine eigenständige, ältere Bildung handelt.

\section{Danksagung}

Wir danken Herrn Prof. Dr. Dr. h.c. A. Semmei für wertvolle Hinweise sowie der Deutschen Forschungsgemeinschaft für eine finanzielle Unterstützung der Arbeiten im Rahmen des Sonderforschungsbereiches 275 „Klimagekoppelte Prozesse in meso- und känozoischen Geoökosystemen" in Tübingen.

\section{Schriftenverzeichnis}

Antoine, P. [Ed.] (1998): Le Quaternaire de la vallée de la Somme et du littoral piccard. - Exc. Assoc. Franç. pour l'Étude du Quaternaire, 21-23 mai 1998, 162 S., 72 Abb.

Antoine, P., Lautridou, J., Sommé, J. et al. (1998): Les formations quaternaires de la France du Nord Ouest: Limités et corrélations. - Quaternaire, 9: 227-241, 3 Abb., 2 Tab., 1 Kte.; Paris.

BeHre, K.-E. \& Lade, U. (1986): Eine Folge von Eem und vier Weichsel-Interstadialen in Oerel/Niedersachsen und ihr Vegetationsablauf. - Eiszeitalter u. Gegenwart, 36: 11-36, 12 Abb., 2 Tab., 2 Taf; Hannover.

Bibus, E., Bludau, W., Bross, C. \& Rähle, W. (1996): Der Altwürm- und Rißabschnitt im Profil MainzWeisenau und die Eigenschaften der Mosbacher Humuszonen. - Frankfurter geowiss. Arb., Serie D, 20: 21-52, 5 Abb., 2 Tab.; Frankfurt a. M.

Caspers, G. \& Freund, H. (1998): Die Vegetationsund Klimaentwicklung des Weichsel-Früh- und Hochglazials im nördlichen Mitteleuropa. - Schriftenr. dt. geol. Ges., 4: 201-249, 31 Abb., 4 Tab.; Hannover.

Cortijo, E., Duplessy, J. C., Labeyrie, L. et al. (1994): Eemian cooling in the Norwegian Sea and North Atlantic ocean preceding continental ice-sheet growth. - Nature, 372: 446-449, 2 Abb.; London.

Dansgaard, W., Johnsen, S. J., Clausen, H. B. et al. (1993): Evidence for general instability of past cli- 
mate from a 250-kyr ice-core record. - Nature, 364: 218-220, 2 Abb.; London.

Evans, J. G. (1972): Land Snails in Archaeology. - 436 S., 145 Abb., 17 Tab.; London, New York.

Field, M.H., Huntley, B. \& Müller, H. (1994): Eemian climate fluctuations observed in a European pollen record. - Nature, 371: 779-783, 3 Abb.; London.

FINK, J. (1954): Die fossilen Böden im österreichischen Löß. - Quartär, 6: 85-108, 11 Abb.; Bonn.

Frechen, M. \& Preusser, F. (1996): Kombinierte Lumineszenz-Datierungen am Beispiel des Lößprofils Mainz-Weisenau. - Frankfurter geowiss. Arb., Serie D, 20: 53-66, 3 Abb., 3 Tab.; Frankfurt a. M.

Gerber, J. (1996): Revision der Gattung Vallonia Risso 1826 (Mollusca: Gastropoda: Valloniidae). - Schriften zur Malakozoologie, 8: 227 S., 85 Abb., 3 Tab.; Cismar.

GRIP (Greenland Ice-Core Project Members) (1993): Climatic instability during the last interglacial period in the GRIP ice core. - Nature, 364: 203-207, 5 Abb., 1 Tab.; London.

LOŽEK, V. (1964): Quartärmollusken der Tschechoslowakei. - Rozpravy Ústr. úst. geol., 31: 374 S., 91 Abb., 32 Taf.; Praha.

Mania, D. (1973): Paläoökologie, Faunenentwicklung und Stratigraphie des Eiszeitalters im mittleren Elbe-Saalegebiet auf Grund von Molluskengesellschaften. - Geologie, 21, Beih., 78/79: 175 S., 14 Abb., 33 Tab.; Berlin.

Mania, D. (1978): Die Molluskenfauna aus den Travertinen von Burgtonna in Thüringen. - Quartärpaläontologie, 3: 59-85, 7 Abb., 2 Tab., 4 Taf.; Berlin.

Manis, D. (1997): Die Molluskenfauna von Wallertheim. - Reports for the Second Wallertheim Workshop, Tübingen, March 23-25, 1997: 46-68, 3 Abb.; Tübingen.

Müller, U. (2000): A Late Pleistocene pollen sequence from the Jammertal in SW-Germany and the influence of altitude on the Eemian vegetation in the Northern Alpine Foreland. - Veget. Hist. Archaeobot., 9: 125-131, 5 fig.; Berlin, Heidelberg.

MünzING, K. (1985): Löß-Boden-Sequenzen am südlichen Oberrhein (Markgräflerland) und ihre Interpretation. - Jh. geol. L.-Amt Baden-Württemberg, 27: 113-143, 5 Abb., 1 Tab.; Freiburg i. Br.

MünzinG, K. \& Löscher, M. (1985): Jungquartäre Molluskenfaunen aus der Umgebung von Heidelberg. - Jh. geol. L.-Amt Baden-Württemberg, 27: 93-112, 3 Abb., 4 Tab.; Freiburg i. Br.

Remy, H. (1968): Zur Stratigraphie und Klimaentwicklung des jüngeren Pleistozäns in Mittel- und Westeuropa unter besonderer Berücksichtigung des Lösses. - Decheniana, 121: 121-145, 4 Abb., 5 Tab.; Bonn.

REMY, H. (1969): Würmzeitliche Molluskenfaunen aus Lößserien des Rheingaues und des nördlichen Rheinhessens. - Notizbl. hess. L.-Amt Bodenforsch., 97: 98-116, 7 Abb., 4 Tab.; Wiesbaden.
Rohdenburg, H. (1964): Ein Beitrag zur Deutung des „Gefleckten Horizontes“. - Eiszeitalter u. Gegenwart, 15: 66-71, 3 Abb.; Öhringen.

Rohdenburg, H. \& Meyer, B. (1966): Zur Feinstratigraphie und Paläopedologie des Jungpleistozäns nach Untersuchungen an südniedersächsischen und nordhessischen Lößprofilen. - Mitt. dt. bodenkdl. Ges., 5: 1-135, 20 Abb.; Göttingen.

RousseAU, D.-D. (1986): Interêt paléobiogéographique de Pupilla loessica Ložek et de Vallonia tenuilabris (A. Braun) pour le Pléistocène ouest-européen. - C. R. Acad. Sc. Paris, Série II, 303: 257-262, 1 Abb.; Paris.

Schönhals, E., Rohdenburg, H. \& Semmel, A. (1964): Ergebnisse neuerer Untersuchungen zur Würmlöß-glieder-ung in Hessen. - Eiszeitalter u. Gegenwart, 15: 199-206, 1 Abb.; Öhringen.

Semmel, A. (1968): Studien über den Verlauf jungpleistozäner Formung in Hessen. - Frankfurter geogr. Hefte, 45: 1-133, 35 Abb., 2 Tab.; Frankfurt a. M.

Semmel, A. (1995): Stop 14: Quarry of the Portlandzementwerke Heidelberg at Mainz-Weisenau. - In: Schirmer, W. [Ed.]: Quaternary field trips in Central Europe, 1: 452-454, 2 Abb.; München.

Semmel, A., [Hrsg.] (1996a): Pleistozäne und holozäne Böden aus Lößsubstraten am Nordrand der Oberrheinischen Tiefebene. Exkursionsführer zur 15. Tagung des Arbeitskreises für Paläopedologie der Deutschen Bodenkundlichen Gesellschaft vom 16. -18.05.1996 in Hofheim am Taunus. - Frankfurter geowiss. Arb., Serie D, 20, 1-144, 25 Abb., 20 Tab.; Frankfurt a. M.

Semmel, A., (1996b): Stop 1, Paläoböden im Würmlöß, insbesondere im Altwürmlöß des Steinbruchs Mainz-Weisenau - Problemstellung und Übersicht über die Forschungsergebnisse.- Frankfurter geowiss. Arb., Serie D, 20: 11-20, 1 Abb., 1 Tab.; Frankfurt a. M.

Semmel, A. (1999a): Die paläopedologische Gliederung des älteren Würmlösses in Mitteleuropa - erörtert an Beispielen aus dem Rhein-Main-Gebiet.- Z. geol. Wiss., 27 (1/2): 121-133, 3 Abb., 2 Tab.; Berlin.

Semmel, A. (1999b): Stop 3: Mainz-Weisenau, Loess section of Mainz-Weisenau.- In: WeIDENFELLER, M. \& Zoeller, L. [Eds.]: Loess in the Middle and Upper Rhine Area, Field Guide: 57-69, 5 Abb., 3 Tab., 3 Fotos; Mainz (Geol. Landesamt). 